\title{
Left atrial flutter due to incomplete left fibrous trigone linear lesion
}

\author{
Laurent Pison, MD, ${ }^{\mathrm{a}}$ Mark La Meir, MD, ${ }^{\mathrm{b}}$ and Harry J. Crijns, MD, PhD, ${ }^{\mathrm{a}}$ Maastricht, The Netherlands
}

From the Department of Cardiology ${ }^{\mathrm{a}}$ and Department of Cardiac Surgery, ${ }^{\mathrm{b}}$ Academic Hospital Maastricht and Cardiovascular Research Institute Maastricht, Maastricht, The Netherlands

Disclosures: Authors have nothing to disclose with regard to commercial support. Received for publication May 26, 2011; revisions received July 10, 2011; accepted for publication July 25, 2011; available ahead of print Sept 12, 2011.

Address for reprints: Laurent Pison, MD, Department of Cardiology, Maastricht University Medical Centre and Cardiovascular Research Institute, Maastricht, PO Box 5800, Maastricht, The Netherlands (E-mail: 1.pison@mumc.nl).

J Thorac Cardiovasc Surg 2011;142:e149-51

$0022-5223 / \$ 36.00$

Copyright $(2011$ by The American Association for Thoracic Surgery doi:10.1016/j.jtcvs.2011.07.050

A

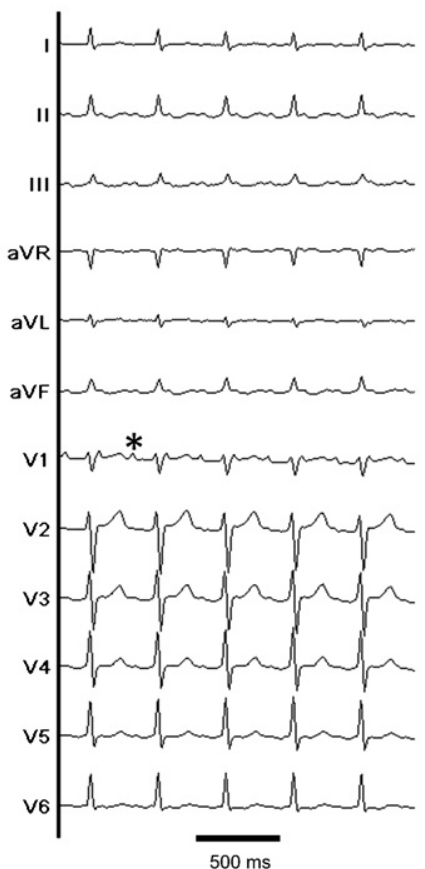

A 59-year-old man underwent minimally invasive thoracoscopic surgical ablation for symptomatic persistent atrial fibrillation (AF) of 7 years' duration. His medication consisted of propafenon, metoprolol, and warfarin. Normal left ventricular systolic function and an enlarged left atrium with a volume of $86 \mathrm{~mL}$ (normal $<66 \mathrm{~mL}$ ) were observed on transthoracic echocardiography. Computed tomography of the coronary arteries revealed no significant stenosis.

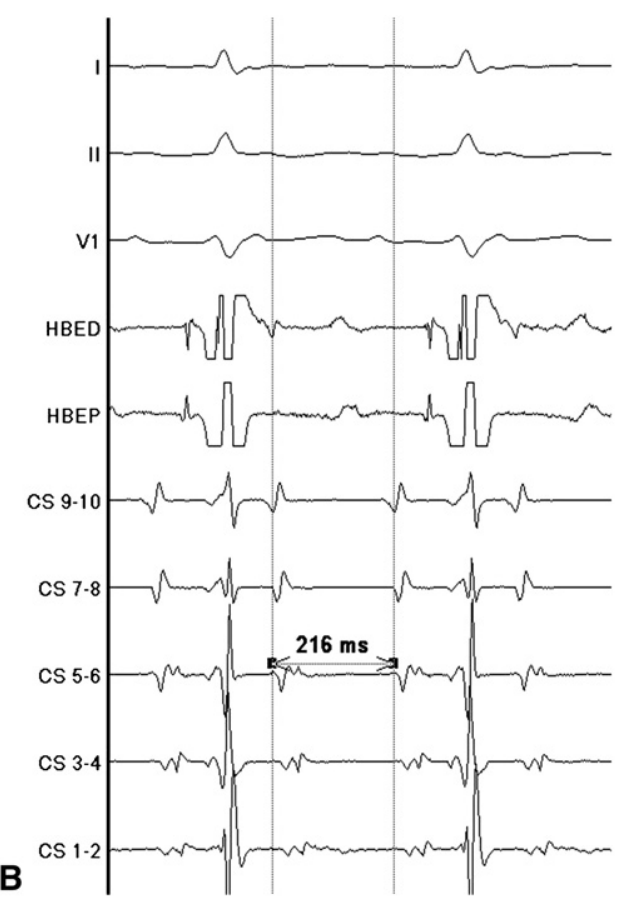

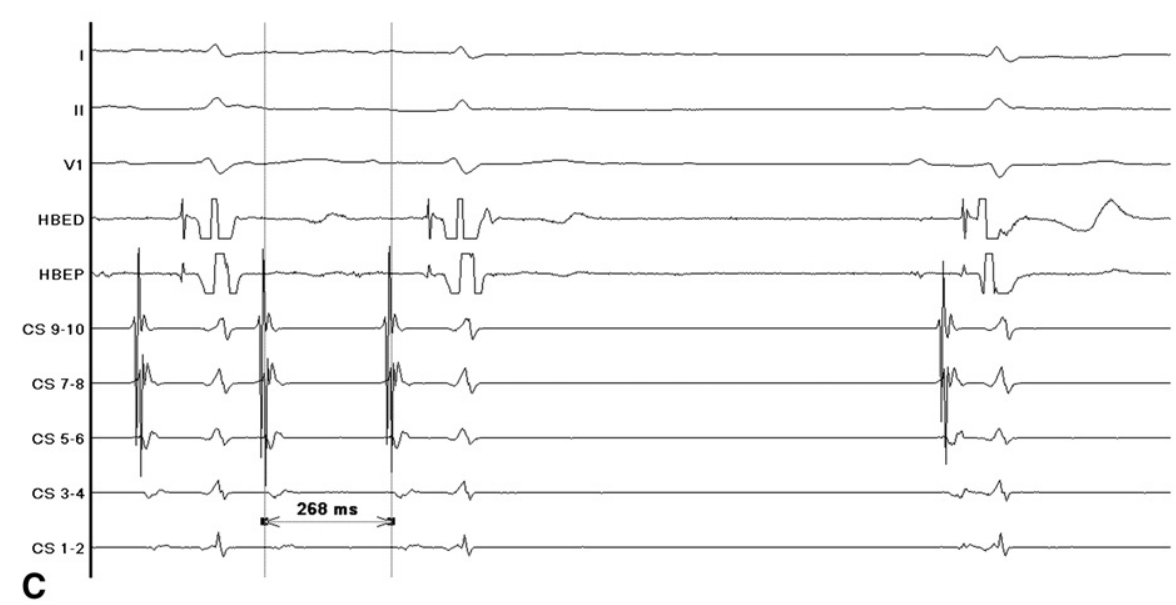

FIGURE 1. A, 12-Lead electrocardiogram showing atrial flutter with positive flutter waves in lead V1 suggesting left atrial origin. B, His ( $H B E)$ and coronary sinus (CS) electrocardiogram showing tachycardia cycle length of $216 \mathrm{~ms}$. C, During endocardial ablation, tachycardia cycle length increased gradually to maximum of $268 \mathrm{~ms}$ and then converted to sinus rhythm when the endocardial linear lesion connected to the roofline. 


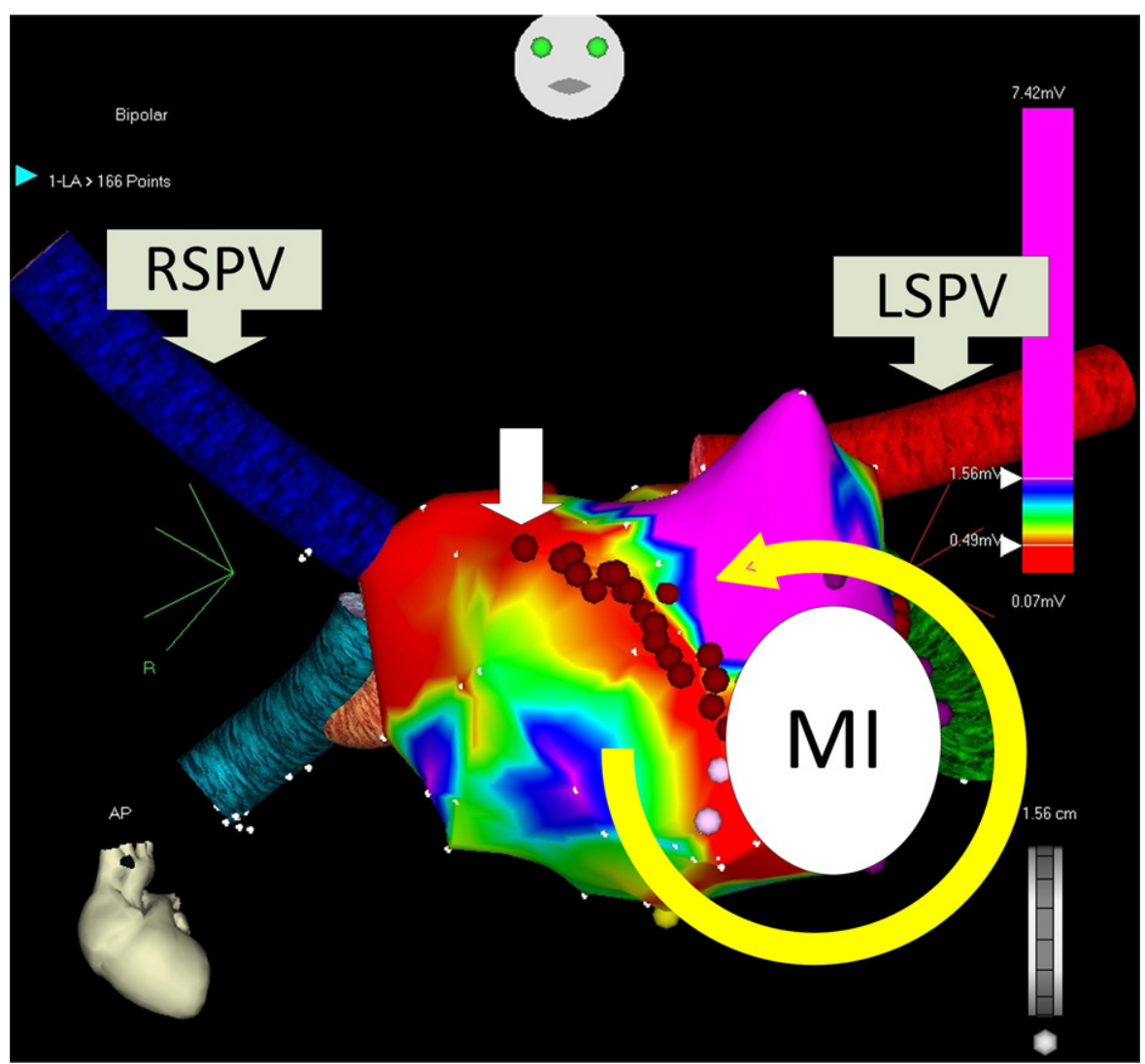

FIGURE 2. Voltage map of left atrium Clinical tachycardia was counterclockwise left atrial flutter evolving around mitral annulus (MI) (yellow arrow). By ablating all the remnant endocardial zones of low voltages at the left fibrous trigone line (red dots), we interrupted surviving strands of atrial tissue, creating a linear lesion extending from the MI to the medial aspect of the roofline (white arrow), resulting in a gradual increase of the tachycardia cycle length and, finally, conversion to sinus rhythm. $L S P V$, left superior pulmonary vein; $R S P V$, right superior pulmonary vein.

During this procedure, the pulmonary veins (PVs) were isolated in pairs using a bipolar radiofrequency clamp (Atricure, West Chester, Ohio). A roofline connecting both superior PVs and an inferior line from one inferior PV to the other were made using a bipolar radiofrequency linear pen device (Coolrail, Atricure). Thus, we created a so-called box lesion. Because of the ongoing AF, a left fibrous trigone line was created using a bipolar radiofrequency pen device (Isolator Pen, Atricure). This connecting lesion was placed from the left fibrous trigone at the anterior mitral valve annulus across the anterior dome of the atrium to the roofline. Because the patient remained in $\mathrm{AF}$ at the end of the procedure, he underwent electrical cardioversion. After an uneventful postoperative recovery, he was discharged with a prescription for warfarin and his preoperative antiarrhythmic drug regimen.

Initially, the patient remained asymptomatic, and the 7-day Holter monitor recordings at 3 and 6 months revealed continuous sinus rhythm. The warfarin and antiarrhythmic drug regimen were stopped. However, 7 months after the procedure, a symptomatic left atrial flutter was documented (Figure 1). Rate control with metoprolol was initiated combined with warfarin. After informed consent, the patient was scheduled for an electrophysiologic study.
After transseptal puncture and placement of a His and a coronary sinus catheter, an activation and endocardial voltage map of the tachycardia was made using a threedimensional electroanatomic mapping system (CARTO, Biosense-Webster, Diamond Bar, Calif; Figure 2). Combined with concealed entrainment mapping, we were able to confirm a counterclockwise left atrial flutter evolving around the mitral valve annulus. By placing a properly sized circular mapping catheter (Lasso, Biosense Webster) at the antrum of the PVs, complete isolation could be proved during tachycardia by the absence of the PV potentials. Also, the box appeared to be isolated, because we detected no nearfield signals on the Lasso catheter placed at the posterior wall of the left atrium. At the endocardial level of the left fibrous trigone line, the voltage map revealed a line of low voltages but no scar extending from the mitral valve annulus toward the medial aspect of the roof line. This indicated that the left fibrous trigone linear lesion created during the surgical ablation procedure was not transmural. Using a 3.5-mmtip catheter (ThermoCool, Biosense Webster), we ablated all the remnant low voltages along this line, starting at the mitral valve annulus (Figure 2). During ablation, we saw a gradual increase in the tachycardia cycle length, and connection of 
the endocardial linear lesion with the roofline resulted in conversion to sinus rhythm (Figure $1, B$ and $C$ ).

Bilateral video-assisted thoracoscopic PV isolation is a safe, beating-heart approach for curative surgical treatment of AF. ${ }^{1}$ Linear lesions are known to improve the outcome of catheter ablation in patients with persistent atrial fibrillation. ${ }^{2}$ The left fibrous trigone line was introduced by Edgerton and colleagues. ${ }^{3}$ This linear lesion serves as an alternative to the endocardial mitral isthmus line extending from the left inferior PV to the mitral valve annulus. The creation of a completely transmural left fibrous trigone line can be hampered by the presence of epicardial fat. Not completely transmural lesions exhibit zones of low voltages and conduction slowing and can become pro-arrhythmic. ${ }^{4}$ In our patient, an incomplete left fibrous trigone line resulted in left atrial flutter. Most of these iatrogenic arrhythmias are very symptomatic. To prevent, as much as possible, such reentry circuits from occurring, it is of paramount importance to prove complete transmurality of each deployed linear lesion. This can effectively be done using an epicardial approach or a combined simultaneous thoracoscopic surgical and transvenous catheter procedure. ${ }^{5}$

\section{References}

1. Wolf RK, Schneeberger EW, Osterday R, Miller D, Merrill W, Flege JB, et al Video-assisted bilateral pulmonary vein isolation and left atrial appendage exclusion for atrial fibrillation. $J$ Thorac Cardiovasc Surg. 2005;130:797-802.

2. Willems S, Klemm H, Rostock T, Brandstrup B, Ventura R, Steven D, et al. Sub strate modification combined with pulmonary vein isolation improves outcome of catheter ablation in patients with persistent atrial fibrillation: a prospective randomized comparison. Eur Heart J. 2006;27:2871-8.

3. Edgerton JR, Jackman WM, Mack MJ. A new epicardial lesion set for minima access left atrial maze: the Dallas lesion set. Ann Thorac Surg. 2009;88:1655-7.

4. Sawhney N, Anousheh R, Chen W, Feld GK. Circumferential pulmonary vein ablation with additional linear ablation results in an increased incidence of left atrial flutter compared with segmental pulmonary vein isolation as an initial approach to ablation of paroxysmal atrial fibrillation. Circ Arrhythm Electrophysiol. 2010;3:243-8.

5. Pison L, La Meir M, Maessen J, Crijns HJ. Hybrid thoracoscopic surgical and transvenous catheter ablation of $\mathrm{AF}$, towards single procedure ablation of longstanding AF. Heart Rhythm. 2010;7(Suppl 5):S398.

\section{Extremely rare but potential complication of diffuse brain edema due to air embolism during lung segmentectomy with selected segmental inflation technique by syringe needle during video-assisted thoracoscopic surgery}

Tsunayuki Otsuka, MD, Yoshihiro Nakamura, MD, PhD, Aya Harada, MD, and Masami Sato, MD, PhD, Kagoshima, Japan

A 65-year-old man was diagnosed with right lung cancer in the upper lobe and S8 segment.

To avoid right pneumonectomy, he underwent right upper lobectomy and S8 segmentectomy by video-assisted thoracoscopic surgery. During segmentectomy, the A8 was ligated first, and then B8 was resected using an automatic stapler. An 18-gauge needle was inserted in the distal B8 bronchus without any blood regurgitation. After removing the inner needle, an air tube of a Bolheal Spray Set (The Chemo-Sero-Therapeutic Research Institute, Kumamoto, Japan) with a filter attached was connected to the outer

\footnotetext{
From the Department of General Thoracic Surgery, Kagoshima University Graduate School of Medical and Dental Sciences, Kagoshima, Japan.

Disclosures: Authors have nothing to disclose with regard to commercial support.

Received for publication July 20, 2011; accepted for publication July 27, 2011; available ahead of print Aug 29, 2011.

Address for reprints: Masami Sato, MD, PhD, Department of General Thoracic Surgery, Graduate School of Medical and Dental Sciences, Kagoshima University, 8-35-1 Sakuragaoka, Kagoshima, 890-8520 Japan (E-mail: m-sato@m2.kufm. kagoshima-u.ac.jp).

J Thorac Cardiovasc Surg 2011;142:e151-2

$0022-5223 / \$ 36.00$

Copyright (c) 2011 by The American Association for Thoracic Surgery doi:10.1016/j.jtcvs.2011.07.061
}

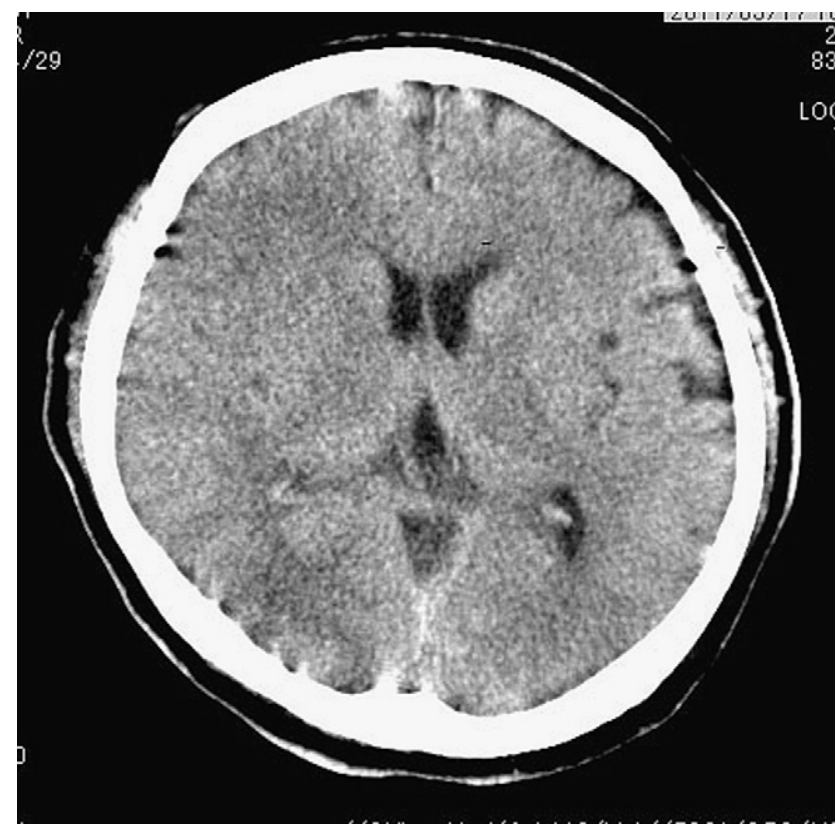

FIGURE 1. Computed tomography scan after hyperbaric oxygen therapy revealing diffuse brain edema. 